\title{
Desenvolvimento de ortorexia nervosa em acadêmicos da sociedade moderna
}

\author{
Development of orthorexia nervosa in academics in modern society \\ Desarrollo de la ortorexia nerviosa en académicos de la sociedad moderna
}

Recebido: 14/03/2021 | Revisado: 21/03/2021 | Aceito: 26/03/2021 | Publicado: 03/04/2021

Hayanne Alanna da Silva Cândido
ORCID: https://orcid.org/0000-0003-0830-7392
Faculdade de Comunicação, Tecnologia e Turismo de Olinda, Brasil
E-mail: hayannecandido@outlook.com
Gleyce Kelly de Araújo Bezerra
ORCID: https://orcid.org/0000-0002-9388-7389
Universidade Federal de Pernambuco, Brasil
E-mail: gleycearaujo.nutri@gmail.com
Maria Suzane da Silva Barbosa
ORCID: https://orcid.org/0000-0003-1995-8721
Universidade Federal de Pernambuco, Brasil
E-mail: m.suzane.barbosa@ gmail.com
Fabio Torres Cunha
ORCID: https://orcid.org/0000-0003-0238-7600
Faculdade de Comunicação, Tecnologia e Turismo de Olinda, Brasil
E-mail: fabiologo1980@gmail.com
Silvia Mariana da Silva Barbosa
ORCID: https://orcid.org/0000-0001-5252-9705
Universidade Federal de Pernambuco, Brasil
E-mail: barbosasms@ gmail.com
Danielle Cássia de Oliveira

\section{Resumo}

Este trabalho teve como objetivo identificar fatores que desencadeiam a ortorexia nervosa, quanto transtorno alimentar, em acadêmicos na sociedade moderna. Para tal, o estudo consistiu de uma revisão com base na análise de artigos no período de 2010 a 2020, nos idiomas inglês, português e espanhol, obtidas nas bases de dados Pubmed, Scielo, Bvs, LILACS e Bireme. A partir da análise de dados, constatou-se que ortorexia nervosa (ON) é mais frequente na população de jovens acadêmicos dos cursos da área da saúde e profissionais da saúde, ressaltando que também acomete indivíduos que possuem extrema preocupação com a imagem física. Concluiu-se que a identificação da Ortorexia é de extrema relevância para prevenir e tratar possíveis quadros patológicos que poderão surgir ao longo do desenvolvimento da ortorexia nervosa quanto transtorno alimentar. É necessário que haja acompanhamento nutricional e psicológico para indivíduos com diagnóstico desse transtorno alimentar. Por se tratar de um transtorno recente, faz-se necessários mais estudos abrangendo a ortorexia de forma minusiosa e íntegra.

Palavras-chave: Comportamento alimentar; Composição corporal; Imagem corporal.

\begin{abstract}
This work aimed to identify factors that trigger orthorexia nervosa, regarding eating disorders, in academics in modern society. To accomplish this the study consisted of a review based on the analysis of articles from 2010 to 2020, in English, Portuguese and Spanish, obtained from the databases Pubmed, Scielo, Bvs, LILACS and Bireme. From the data analysis, it was found that Orthorexia nervosa $(\mathrm{ON})$ is more frequent in the population of young academics in health courses and health professionals, emphasizing that it also affects individuals who are extremely concerned with physical image. It was concluded that the identification of Orthorexia is extremely important to prevent and treat possible pathological conditions that may arise during the development of orthorexia nervosa regarding eating disorders. Nutritional and psychological monitoring is required for individuals diagnosed with this eating disorder. As it is a recent disorder, further studies are needed, covering orthorexia in a thorough and thorough manner.
\end{abstract}

Keywords: Feeding Behavior; Body composition; Body image.

\section{Resumen}

Este trabajo tuvo como objetivo identificar los factores desencadenantes de la ortorexia nerviosa, en relación con los trastornos alimentarios, en académicos de la sociedad moderna. Para tal, el estudio consistió en una revisión basada en 
el análisis de artículos de 2010 a 2020, en inglés, portugués y español, obtenidos de las bases de datos Pubmed, Scielo, Bvs, LILACS y Bireme. Del análisis de datos, la ortorexia nerviosa $(\mathrm{ON})$ es más frecuente en la población de jóvenes académicos en cursos de salud y profesionales de la salud, destacando que también afecta a individuos extremadamente preocupados por la imagen física. Conclusión: La identificación de la Ortorexia es de suma importancia para prevenir y tratar las posibles condiciones patológicas que pueden surgir durante el desarrollo de la Ortorexia nerviosa en relación con los trastornos alimentarios. Se requiere un control nutricional y psicológico para las personas diagnosticadas con este trastorno alimentario. Como es un trastorno reciente, se necesitan más estudios que cubran la ortorexia de manera minuciosa y completa.

Palabras clave: Conducta alimentaria; Trastorno alimentario; Nutrición.

\section{Introdução}

Os transtornos alimentares (TA) são representados por uma perturbação incessante na alimentação ou no comportamento relacionado à alimentação que resulta no consumo ou na absorção modificada de alimentos e que compromete significativamente a saúde física ou psicológica (Vital, Silva, Garcia \& Omena, 2017).

A ortorexia nervosa $(\mathrm{ON})$ vem sendo apontada na ciência como um novo tipo de anormalidade do comportamento alimentar, do tipo obsessivo-patológico, no sentido de preocupação exagerada com a qualidade dos alimentos e pureza da dieta. O termo "ortorexia" significa "alimentação correta" e é oriundo do grego "orto", que significa "correto, direito", e "rexia", referindo-se a apetite (Penaforte et al. 2018).

Pessoas com esse transtorno evitam severamente a ingestão de alimentos devido ao seu conteúdo em gordura, conservantes, aditivos ou outros componentes considerados deletérios ou tóxicos. Essas apreenções os levam a uma vida determinada pela comida, gerando deficiências em áreas sociais, familiares e/ou ocupacionais (Barrada \& Roncero, 2018).

A vida interior do indivíduo com ortorexia é determinada por esforços para não ser tentado a ingerir alimentos não saudáveis. Da mesma forma, aqueles que sofrem de ON podem atingir um estado de desnutrição, através da exclusão de certos nutrientes, com as várias consequências que isso pode causar, por exemplo, a alteração do sistema imunológico (Cavero et al. 2015).

Esse transtorno alimentar acomete cerca de 0,5 a $1 \%$ da população, entre os dois sexos com mais frequência, particularmente em artistas, desportistas, médicos e graduandos de medicina, jovens e pessoas que são cientes da grande importância da nutrição correta para a manutenção da saúde (Potyrala \& Olejniczak, 2018).

Na patogenia da ON, estão envolvidos fatores genéticos, psicológicos, sociais, culturais, nutricionais, neuroquímicos e hormonais, atuando como predisponentes desencadeadores ou mantenedores da alteração. Destaca-se o impacto da mídia e, principalmente, da televisão nesses distúrbios alimentares, servindo como forma de propagação do "estereótipo social" e dos comportamentos e hábitos de vida (Cuquejo et al. 2017).

$\mathrm{Na}$ atuação clínica é empregado o teste de rastreamento da ortorexia nervosa, Orto-15, uma ferramenta que descreve a intensidade do comportamento do ortoréxico. E sucessivamente o indivíduo deve ser acompanhado por uma equipe multiprofissional. Onde a conduta nutricional recomendada é uma dieta balanceada, juntamente com terapias cognitivocomportamentais (Potyrala \& Olejniczak, 2018).

O nível socioeconômico elevado também está associado ao desenvolvimento desse transtorno, visto que os alimentos considerados puros geralmente são encontrados com preço elevado, fazendo com que esse distúrbio seja mais suscetível em países desenvolvidos (Rocha et al. 2016).

Ressaltando que algumas variáveis pode afetar a imagem corporal de jovens acadêmicos, como por exemplo, o curso de graduação em que o sujeito está matriculado. Existe uma crença de que estudantes do curso de Educação Física e Nutrição sejam aqueles que apresentam níveis mais altos de insatisfação corporal e maior risco para o surgimento de transtornos alimentares (Oliveira et al. 2017). 
Nesse contexto, o principal objetivo desse estudo foi identificar os fatores para o desenvolvimento da ortorexia enquanto transtorno na alimentação em jovens acadêmicos.

\section{Metodologia}

O presente estudo consiste em uma revisão de literatura quantitativa sobre as causas e efeitos da Ortorexia, com ênfase em jovens acadêmicos. Com análise de artigos obtidos das seguintes bases de dados: Scientific Eletronic Library Online (Scielo), National Library of Medicine (Pubmed), Biblioteca Virtual em Saúde (Bvs), Literatura Latino-Americana e do Caribe em Ciências da Saúde (LILACS/Bireme). Para identificar publicações relevantes, foram realizadas buscas nas bases de dados do período de 2010 a 2020.

A estratégia de busca foi definida por descritores cadastrados nos Descritores em Ciências da Saúde (DECs) com termos relativos a comportamento alimentar (ortorexia, transtornos alimentares, nutrição).

Os critérios de inclusão foram definidos previamente; artigos redigidos nos idiomas português, inglês e espanhol; artigos na versão completa, teses de doutorado e mestrado e revisão; estudos realizados em acadêmicos e adolescentes, publicados nos últimos dez anos. Os critérios de exclusão de artigos compreenderam: artigos não disponíveis na versão completa, comunicações breves, TCC (trabalho de conclusão de curso) e relacionados ao surgimento da ortorexia em outras faixas etárias.

Os artigos foram avaliados quanto à principal questão da pesquisa: desenvolvimento da ortorexia em acadêmicos. Todos os artigos que se enquadravam no presente estudo foram incluídos.

\section{Resultados}

O resultado da busca nas bases de dados, descritos no fluxograma da Figura 1, resultou em: vinte e seis (26) artigos no total. Dos 26 artigos iniciais, 01 era duplicado e foi excluído da análise. Dos 25 artigos restantes, foi realizada uma triagem por meio de leitura do título e resumo e foram excluídos 3, por estarem enquadrados nos critérios de exclusão. Deste modo, vinte e dois artigos (22) integram esta revisão.

Após identificação dos artigos que cumpriram os critérios para inclusão, vinte e dois (22) artigos selecionados, estes foram analisados e em seguida realizada a extração dos dados para a composição deste estudo vide Tabela 1. 
Figura 1. Fluxograma da pesquisa dos artigos.

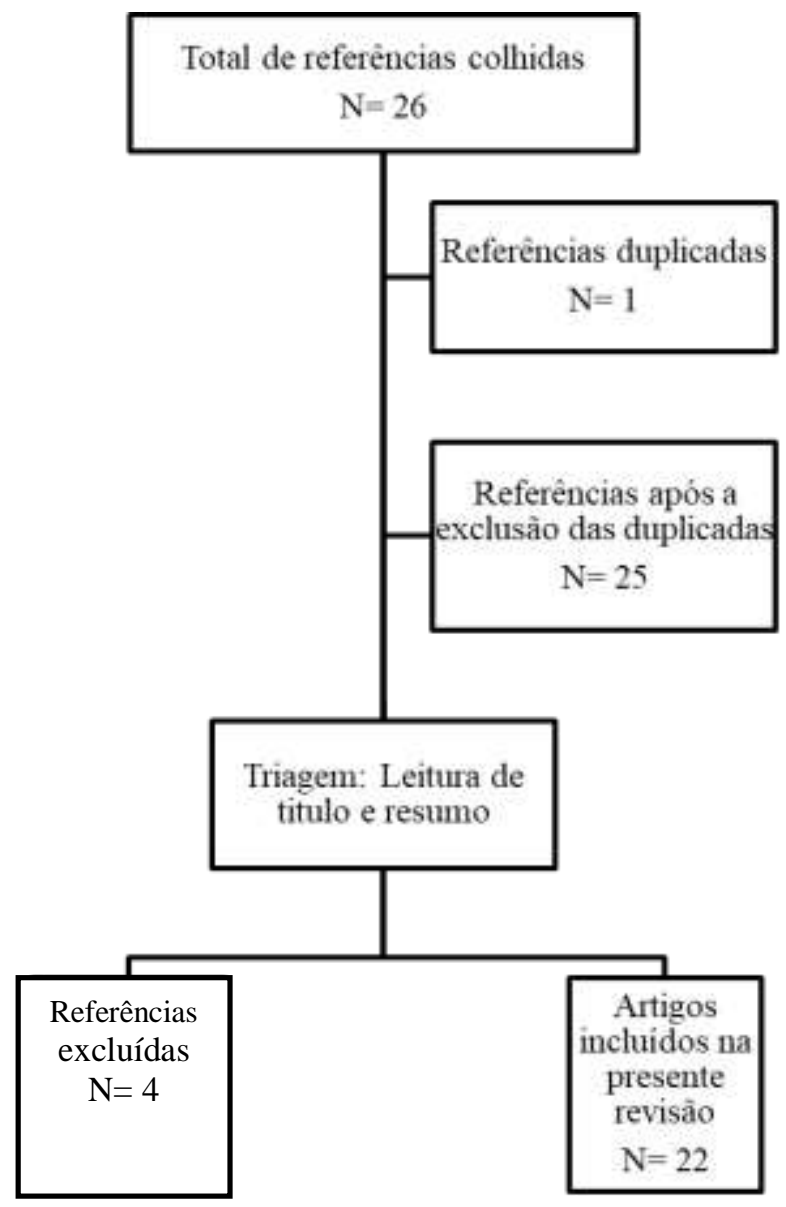

Fonte: Autores.

Tabela 1. Objetivo, resultado e conclusão dos autores reunidos no presente estudo.

\begin{tabular}{|c|c|c|c|}
\hline Autor/ ano & Objetivo & Resultados & Conclusão \\
\hline $\begin{array}{l}\text { Plichta, } \\
\text { et al; } 2020\end{array}$ & $\begin{array}{l}\text { Explorar padrões dietéticos e } \\
\text { outros hábitos alimentares de } \\
\text { pessoas com tendência a } \\
\text { ortorexia nervosa e sintomas } \\
\text { de transtornos alimentares. }\end{array}$ & $\begin{array}{l}\text { Participantes que afirmaram ter feito } \\
\text { uso de dietas no passado, atualmente } \\
\text { aumentaram a tendência para ON. Os } \\
\text { alunos que apresentavam ON e TA } \\
\text { foram caracterizados pelo consumo } \\
\text { menos frequente de açúcar e produtos } \\
\text { refinados. E os que menos consumiam } \\
\text { carne demonstraram ser menos } \\
\text { propensos a ON e TA. }\end{array}$ & $\begin{array}{l}\text { Diferentes hábitos alimentares de } \\
\text { pessoas com tendência a ON e } \\
\text { TA confirmou a diferença entre } \\
\text { ON e TA. Porém a exibição } \\
\text { simultânea de ambos leva a } \\
\text { sobreposição da especificidade de } \\
\text { hábitos alimentares o que } \\
\text { dificulta no diagnóstico. }\end{array}$ \\
\hline $\begin{array}{l}\text { Medina- Goméz, } \\
\text { et al; } 2019\end{array}$ & $\begin{array}{l}\text { Analisar os níveis de ansiedade } \\
\text { e satisfação corporal e suas } \\
\text { relações com o gênero. }\end{array}$ & $\begin{array}{l}20 \% \text { dos participantes apresentaram } \\
\text { altos níveis de ansiedade, não tendo } \\
\text { diferença entre sexo. Entretanto, a } \\
\text { maior taxa de insatisfação corporal } \\
\text { ocorreu nas mulheres. }\end{array}$ & $\begin{array}{l}\text { Conclui-se que a insatisfação } \\
\text { corporal e } \\
\text { relacionam-se }\end{array}$ \\
\hline
\end{tabular}


Cruz,

et al; 2018

Silva,

et al; 2018

Kessler, et al; 2018

Mazzia, et al; 2018

Marchi, et al; 2018

Scholl, et al; 2017

Coelho, et al; 2016

Silveira Jr, et al; 2015
Verificar por meio da anamnese alimentar o perfil de estudantes de nutrição com tendências ao quadro de ortorexia nervosa $(\mathrm{ON})$.

Verificar as relações entre imagem corporal, estresse, autoestima, comportamento alimentar e índice de massa corporal em adolescentes e jovens, respondendo a testes e inventários.

Avaliar a relação entre a insatisfação da imagem corporal, atitudes para transtornos alimentares e o estado nutricional em universitárias da área da saúde.

Identificar a presença de fatores de risco para transtornos alimentares em estudantes de enfermagem para o desenvolvimento de transtornos alimentares.

Verificar a presença de ortorexia nervosa em estudantes do curso de nutrição de uma instituição de ensino superior no sudoeste do Paraná.

Avaliar a qualidade de vida em portadores de TOC (transtorno obsessivo compulsivo) usuários da atenção primária de saúde.

Conceituar a ortorexia nervosa caracterizando-a; apontar os sintomas e os motivos da baixa dimensão dos estudos acerca da referida patologia; e, especificamente, abrangendo os efeitos da doença.

Expor, através de uma revisão bibliográfica, evidências que relacione a ortorexia nervosa ao transtorno obsessivocompulsivo.
Num estudo realizado com estudantes de nutrição de ambos os sexos, observou que $71 \%$ encontraram-se eutróficos, $80 \%$ com circunferência abdominal dentro do esperado e $71 \%$ com tendência ao quadro de ortorexia.

Meninas apresentaram riscos para distúrbios alimentares, maior preocupação com IC, mais estresse e menor autoestima e meninos desejam ser mais musculosos através de análises.

Segundo o estudo realizado, a maioria das universitárias se encontraram em eutrofia, onde a maioria gostaria de pesar menos. Houve prevalência de atitudes indicativas de transtornos alimentares.

Foi identificado dentre os 120 graduandos a presença de alteração de comportamento alimentar e insatisfação com a imagem corporal. Colocando em evidência que os graduandos mais jovens tendem a apresentar maior risco para transtornos alimentares.

O estudo envolvendo 82 alunos, sendo 77 do sexo feminino e 5 do masculino, resultou em 66 estudantes do sexo feminino com predisposição a desenvolvimento ortorexico e todos do sexo masculino também.

$\mathrm{Na}$ amostra de 1081 indivíduos avaliados a prevalência de TOC foi de $3,9 \%$.

Uma alimentação seletiva que pode gerar a carência de determinados nutrientes no organismo, implicando diversas consequências, essas podendo ser físicas, psíquicas e/ou sociais.

Não foram encontrados estudos experimentais que demonstrassem relação direta entre ortorexia nervosa e transtono obsessivo- compulsivo.
Por se tratar de estudantes de nutrição que buscam uma alimentação saudável, faz-se necessário uma atenção maior para que esse comportamento não se torne obsessivo e gere um quadro de ortorexia.

Foram identificadas relações que podem subsidiar intervenções preventivas e centradas em variáveis, como a preocupação em emagrecer, estresse e autoestima em meninas, e o desejo de aumentar a massa corporal nos meninos.

Evidenciou-se a relação entre a insatisfação com a imagem corporal, atitudes de risco para transtornos alimentares e estados nutricional de acadêmicas da área da saúde.

Graduandos de enfermagem apresentam risco para desenvolvimento de transtornos alimentares e sugere que pesquisas focalizem na prevenção, no conceito positivo sobre autoimagem.

Com resultados positivos para o desenvolvimento sugere-se que novas pesquisas sejam realizadas a fim de traçar o perfil da população brasileira pra prevalência do comportamento.

O estudo mostra a importância da avaliação da qualidade de vida como instrumento para melhora do transtorno no âmbito da atenção básica à saúde.

Entende-se que existem formas de prevenir a ortorexia por meio da psicoedução e transmissão de informação correta, visto que é um comportamento alimentar recente.

Salienta a necessidade de estudos sobre o tema, pois a relação entre transtornos interferem em seus diagnósticos, prognósticos e na elaboração de estratégias terapêuticas. 


$\begin{array}{ll}\text { Brytek-Matera, } & \begin{array}{l}\text { Explorar as relações entre as } \\ \text { características da ortorexia e ás }\end{array} \\ \text { atitudes em relação a imagem } \\ \text { et al; } 2015 & \begin{array}{l}\text { corporal, aptidão e saúde em } \\ \text { universitários. }\end{array}\end{array}$

Identificar comportamento de Souza, risco para o desenvolvimento et al; 2014 de ortorexia nervosa em estudantes de Nutrição.

Petroski, et al; 2012

Verificar os motivos e a prevalência de insatisfação com a imagem corporal (IC) em adolescentes.
Não houve diferenças significativas em relação aos níveis de comportamento de ortorexia entre mulheres e homens. Em relação à aparência, segundo o estudo, foi significativamente relevante em mulheres.

Entre 150 alunas avaliadas, verificou-se que 111 estavam eutróficas, $112 \mathrm{em}$ relação ao total apresentava distúrbio de imagem e 113 em relação ao total apresentavam riscos para desenvolver ortorexia.

A prevalência de insatisfação com índice corporal foi de 60,4\% numa amostra de 641 adolescentes. Onde a maior prevalência foi em adolescentes urbanos e autoestima por adolescentes rurais.

O estudo identificou grupos vulneráveis para desenvolvimento da ortorexia: estudantes de medicina, médicos, nutricionistas, pessoas com ansiedade, obsessivo-compulsivos e aqueles que supervalorizam o corpo perfeito.
Uma forte preocupação com uma alimentação saudável não foi associada a uma relação doentia corpo-self entre estudante polonesa com ortorexia nervosa.

Constatou-se que um número considerável de alunas apresentavam comportamento ortoréxico e distúrbio de imagem pessoal.

A estética, autoestima e saúde são motivos que mais influenciam com a insatisfação do índice corporal (IC). Nesse sentido, intervenções por profissionais são necessárias para prevenir problemas futuros.

Certifica que a ortorexia é situada a partir de uma análise de conceitos de atitudes alimentares, procurando foco psicossocial e fisiológico.

Fonte: Autores.

\section{Discussão}

A prevalência de insatisfação com a imagem corporal é maior em indivíduos do sexo feminino do que no sexo masculino. Onde as moças desejam diminuir a silhueta corporal e os rapazes desejam aumentar segundo o estudo avaliado por (Petroski et al. 2012). Em contrapartida, em (Medina-Goméz et al. 2019) seu estudo transversal aplicado numa amostra de estudantes de ambos os sexos não foi encontrada diferença significativa entre insatisfação corporal e ansiedade por classificação de sexo, porém, no que diz respeito a insatisfação corporal, o sexo feminino demonstrou maiores níveis.

De acordo com (Souza \& Rodrigues, 2014) na pesquisa realizada com universitárias do curso de nutrição verificou-se que $88,7 \%$ apresentaram risco para desenvolvimento de ortorexia nervosa, embora $74 \%$ foram encontradas com estado nutricional de eutrofia. Convém ao estudo realizado por (Cruz et al. 2018) que em uma amostra de estudantes de ambos os sexos do curso de nutrição de uma determinada instituição, foi possível observar que a maior parte da população do estudo encontra-se eutróficos.

(Silva et al. 2018) explana em seu estudo amostral que o grau de insatisfação com a imagem corporal foi significativamente maior em indivíduos do sexo masculino, que possuíam o desejo de engordar. Já (Kessler \& Poll, 2019) afirma em seu estudo descritivo transversal que ao relacionar insatisfação com a imagem corporal e atitudes de risco para desenvolvimento de transtornos alimentares, percebeu que 87,75\% das universitárias com EAT positivo (Eating Attitudes Test) também apresentaram insatisfação corporal.

No estudo quantitativo, (Mazzia \& Santos, 2018) destaca que em sua amostra com 120 participantes, 30 apresentaram fatores de risco para o desenvolvimento de transtornos alimentares e 18 apresentaram alterações no IMC (índice de massa corpórea). Porém, (Marchi \& Baratto, 2018) ao relacionar os valores de IMC de uma amostra de 82 acadêmicos, com a 
presença de sintomas para ortorexia verificou-se que os indivíduos com maior risco para o desenvolvimento são aqueles que apresentam valores de IMC menores.

Silveira et al. (2015) em seu estudo ocorre a relação entre a ortorexia e o transtorno obsessivo compulsivo (TOC), afirmando que a ortorexia, apesar de ser considerada um transtorno alimentar, se assemelha ao transtorno obsessivo quando o individuo passa a ter uma busca excessiva por uma dieta saudável e livre de impurezas. (Scholl et al. 2017) expõe em seu estudo que indivíduos com TOC tiveram uma redução significativa de domínio físico, psicológico e sociais comparados a indivíduos sem TOC o que trás sentido a essa associação da ortorexia com o transtorno obsessivo compulsivo.

De acordo com (Coelho et al. 2016) a ortorexia surge como um desejo de melhorar a saúde do indivíduo, tratar uma doença ou emagrecer, mas com o passar do tempo acaba se tornando algo obsessivo. (Martins et al. 2011) relaciona ortorexia com distúrbio de personalidade ou comportamento psicológico declarando ter ligação entre as relações de crenças e atitudes ligadas a religião ou filosofia de cada indivíduo, já que muitas religiões valorizam práticas alimentares ascéticas.

(Brytek-matera et al. 2015) em seu estudo amostral em uma análise de regressão, concluiu que há baixa satisfação das áreas corporais, orientações fitness, preocupação com sobrepeso e orientação de aparência foram preditivos na forte preocupação em relação à alimentação saudável entre mulheres.

Conforme o estudo amostral realizado por (Plichta \& Jezewska-zychowicz, 2020) foi concluído que os alunos que mantinham o intervalo de mais de 4 horas entre as refeições de forma irregular tinham menos propensão a desenvolver ON do que os alunos que faziam a refeição a cada 3 horas de forma regular. O mesmo encontrou também, alguns alunos que raramente consumiam alimentos com alto teor de açúcar e produtos refinados eram menos propensos a exibir ortorexia nervosa.

\section{Considerações Finais}

Através do presente estudo foi possível constatar que a ortorexia nervosa $(\mathrm{ON})$ acomete principalmente jovens acadêmicos e indivíduos cientes da importância de uma alimentação adequada e saudável. Onde esse transtorno ocasiona no individuo danos que poderão resultar em problemas nutricionais e psíquicos.

Do ponto de vista nutricional, é de extrema importância que indivíduos com sinais de ortorexia nervosa busquem acompanhamento com um profissional nutricionista para o diagnóstico com intervenção e acompanhamento psicológico, visando à manutenção da saúde. Por se tratar de um transtorno recente, o presente estudo encontrou limitações durante as buscas de artigos relacionados ao tema.

Portanto, faz-se necessários mais estudos de investigação, estudo de caso, revisões bibliográficas envolvendo questões da ortorexia nervosa em um âmbito geral, incluindo vários níveis populacionais devido ao aumento dos casos, abrangendo assim esse referido distúrbio de uma forma mais completa.

\section{Referências}

Barrada, J. R., \& Roncero, M. (2018). Bidimensional structure of the orthorexia: Development and initial validation of a new instrument. Anales De Psicología/Annals of Psychology, 34(2), 283-291. https://doi.org/10.6018/analesps.34.2.299671

Brytek-Matera, A., Donini, L. M., Krupa, M., Poggiogalle, E., \& Hay, P. (2015). Orthorexia nervosa and self-attitudinal aspects of body image in female and male university students. Journal of Eating Disorders, 3(1), 2. https://doi.org/10.1186/s40337-015-0038-2

Cavero, V., Lodwig, C., Lulli, G., \& Tejada, R. (2015). La ortorexia: Un nuevo reto para los profesionales de la salud. ISSN: 0004-0622

Cruz, R. T., Souza, C. T., Francisqueti, F. V., \& Souza, D. T. (2018). Verificação do estado nutricional de estudantes do curso de Nutrição das Faculdades Integradas de Bauru-SP com enfoque na ortorexia. RBONE-Revista Brasileira De Obesidade, Nutrição E Emagrecimento, 12(76), 1119-1128. ISSN: ISSN 1981-9919 
Coelho, G. C., Troglio, G. M., Hammes, L., Galvão, T. D., \& Cyrino, L. A. R. (2016). As consequências físicas, psíquicas e sociais em indivíduos com ortorexia nervosa. RBONE - Revista Brasileira De Obesidade, Nutrição E Emagrecimento, 10(57), 160-168. http://www.rbone.com.br/index.php/rbone/article/view/408

Cuquejo, L. M. O., Aguiar, C., Domínguez, G. C. S., \& Hermosilla, A. T. (2017). Transtornos alimentares em adolescentes: uma patologia em crescimento? Pediatrics (Asunción), 44 (1), 37-42. https://doi.org/10.18004/ped.2017.abril.37-42

Kessler, A. L., \& Poll, F. A. (2018). Relação entre imagem corporal, atitudes para transtornos alimentares e estado nutricional em universitárias da área da saúde. Jornal Brasileiro de Psiquiatria, 67(2), 118-125. https://doi.org/10.1590/0047-2085000000194

Marchi, P. de, \& Baratto, I. (2018). Prevalência de ortorexia nervosa em acadêmicos do curso de Nutrição em uma Instituição de Ensino Superior no sudoeste do Paraná. RBONE - Revista Brasileira De Obesidade, Nutrição E Emagrecimento, 12(74), http://www.rbone.com.br/index.php/rbone/article/view/771

Martins, M. C. T., Alvarenga, M. dos S., Vargas, S. V. A., Sato, K. S. C. de J., \& Scagliusi, F. B. (2011). Ortorexia nervosa: reflexões sobre um novo conceito. Revista de Nutrição, 24(2), 345-357. https://doi.org/10.1590/S1415-52732011000200015

Mazzaia, M. C., \& Santos, R. M. C. (2018). Fatores de risco para transtornos alimentares em graduandos de enfermagem. Acta Paulista de Enfermagem, 31(5), 456-462. https://doi.org/10.1590/1982-0194201800065

Medina-Gómez, M. B., et al (2019). Ansiedad e insatisfacción corporal en universitarios. Acta Colombiana de Psicología,22(1), 13-21. https://dx.doi.org/10.14718/acp.2019.22.1.2

Oliveira, P. L. D., Ferreira, M. E. C., Neves, C. M., Meireles, J. F. F., \& Carvalho, P. H. B. D. (2017). Insatisfação, checa gem corporal e comportamentos de risco para transtornos alimentares em estudantes de cursos da saúde. Jornal Brasileiro de Psiquiatria, 66(4), 216-220. http://dx.doi.org/10.1590/00472085000000174

Penaforte, F. R. O., et al (2018). Ortorexia nervosa em estudantes de nutrição: associações com o estado nutricional, satisfação corporal e período cursado. Jornal Brasileiro de Psiquiatria, 67(1), 18-24. https://doi.org/10.1590/0047-2085000000179

Petroski, E. L., Pelegrini, A., \& Glaner, M. F. (2012). Motivos e prevalência de insatisfação com a imagem corporal em adolescentes. Ciência \& Saúde Coletiva, 17(4), 1071-1077. https://doi.org/10.1590/S1413-81232012000400028

Plichta, M., \& Jezewska-Zychowicz, M. (2020). Orthorexic tendency and eating disorders symptoms in Polish students: examining differences in eating behaviors. Nutrients, 12(1), 218. https://doi.org/10.3390/nu12010218

Potyrała, P., \& Olejniczak, D. (2018). Nonspecific eating disorders: ortorexia nervosa and night eating syndrome-a subjective review. Journal of Education, Health and Sport, 8(9), 301-307. http://dx.doi.org/10.5281/m9.figshare.6983972.

Rocha, M. A. P. da (2016). Ortorexia: uma compulsão por alimentos saudáveis. Nutrição Brasil, 14(1). ISSN: 2526-7779

Scholl, C. C., Tabeleão, V. P., Stigger, R. S., Trettim, J. P., Mattos, M. B. D., Pires, A. J., \& Quevedo, L. D. A. (2017). Qualidade de vida no Transtorno Obsessivo-Compulsivo: um estudo com usuários da Atenção Básica. Ciência \& Saúde Coletiva, 22, 1353-1360. https://doi.org/10.1590/141381232017224.02062015 .

Silva, A. M. B. da, et al (2018). Jovens Insatisfeitos com a Imagem Corporal: Estresse, Autoestima e Problemas Alimentares. Psico-USF, 23(3), 483495. https://dx.doi.org/10.1590/1413-82712018230308

Silveira Jr, L. A. da S., et al (2015). Ortorexia nervosa e transtorno obsessivo-compulsivo: qual a relação? Psicologia Hospitalar, 13(2), 47-63. http://pepsic.bvsalud.org/scielo.php?script=sci_arttext\&pid=S167774092015000200004\&lng=pt\&tlng=pt.

Souza, Q. J. O. V. D., \& Rodrigues, A. M. (2014). Comportamento de risco para ortorexia nervosa em estudantes de nutrição. Jornal brasileiro de psiquiatria. $10.1590 / 0047-2085000000026$

Vital, A. N. S., Silva, A. B. A., Garcia, E. I., \& de Omena Messias, C. M. B. (2017). Risco para desenvolvimento de ortorexia nervosa e o comportamento alimentar de estudantes universitários. Saúde e Pesquisa ISSN 2176-9206, 10(1), 83-89. http://dx.doi.org/10.177651/1983-1870.2017v10n1p83-89 